Publicación semestral. ISSN 2215-4906

Volumen 79 - Número 1

Julio - Diciembre 2019

El cine para enseñar y aprender Historia del Arte.

La pintura de William Turner

Cinema to Teach \& Learn Art History.

The Painting of William Turner

Ethan Calero Ortuño

Juan Ramón Moreno-Vera

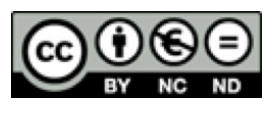

Esta obra está bajo una licencia Creative Commons

Reconocimiento-No comercial-Sin Obra Derivada 


\title{
El cine para enseñar y aprender la historia del arte. La pintura de William Turner
}

\author{
Cinema to teach \& learn art history. \\ The painting of William Turner
}

\author{
Ethan Calero Ortuño ${ }^{1}$ \\ Universidad de Murcia \\ España \\ Juan Ramón Moreno-Vera² \\ Universidad de Murcia \\ España
}

Recibido: 29 de agosto del 2018 Aprobado: 26 de noviembre del 2018

\begin{abstract}
Resumen
La investigación que aquí se presenta tiene por objeto analizar los resultados de aprendizaje de una experiencia didáctica en la que se ha utilizado el cine, en concreto, la película Mr. Turner (dirigida por Mike Leigh en 2014) para aprender las características de la pintura del Romanticismo con estudiantes de segundo curso de Bachillerato. La metodología empleada, poco frecuente todavía en la enseñanza del arte, ha mostrado unos resultados muy positivos en la adquisición de conocimiento por parte del alumnado, tanto para analizar la personalidad del autor como para comprender su pintura y también el mercado del arte en el que se movían los pintores de la época.
\end{abstract}

Palabras clave: Cine; Historia del arte; tableau vivant; Romanticismo; Turner.

1 Becario pre-doctoral. Historiador, máster en Formación del Profesorado por la Universidad de Alicante, España. Correo electrónico: ethan_21_@hotmail.com

2 Profesor en la Universidad de Murcia, España. Doctor en investigación educativa por la Universidad de Alicante, España. Correo electrónico: jr.moreno@um.es 


\begin{abstract}
The paper presented has as target the analysis of the results obtained in a didactic action developed using the film Mr. Turner (Mike Leight, 2014). In this action, students had to learn the caractheristics of the romantic painting through the film. The methodology used, still infrequent in art education, shows a very positive result in knowledge acquisition by the students in order to learn the author's personality, his style and the art market of that moment.
\end{abstract}

Keywords: Cinema; History of art; tableau vivant; romanticism; Turner 


\section{Introducción}

En el presente trabajo se presentará una propuesta de innovación didáctica a través del cine, para trabajar el Romanticismo británico insertado dentro de la asignatura de Historia del Arte de segundo año de bachillerato. Para ello, se procederá a enumerar los diversos métodos que podemos seguir a la hora de trabajar con el cine en el contexto del aula, así como los objetivos y criterios por los que hemos seleccionado el filme de Mike Leigh, Mr. Turner (2014), sobre la vida del pintor británico Joseph Mallord William Turner. Al mismo tiempo, presentaremos los medios de evaluación que hemos aplicado para comprobar si el cine es un recurso útil a la hora de acercar la Historia del Arte a los estudiantes.

Durante décadas, se ha utilizado el cine como recurso didáctico, siempre con ciertas dificultades -asociadas a los problemas técnicos de su introducción en las aulas-, pero con cierto éxito, cuando la selección de las películas estaba ajustada a la edad de los estudiantes, a los contenidos que debían aprender y a la forma de recrear que tenían las propias cintas. En este sentido, en la didáctica de la historia, ha sido un recurso utilizado con acierto. A pesar de esto, en los contenidos de Historia del Arte ha sido un recurso mucho menos utilizado, tal vez por las características de la asignatura (en el currículum español se encuentra en el último curso de Bachillerato, previo al examen de acceso a la universidad, por lo que el profesorado se concentra en transmitir el contenido, pero no atiende a innovaciones metodológicas), por lo amplio del temario que impide detenerse y dedicar tiempo a cada una de las etapas, o tal vez porque los propios contenidos de la Historia del Arte no están muy desarrollados en el medio cinematográfico.

Así pues, a pesar de que el empleo de filmes para trabajar hechos históricos se lleva a cabo desde hace décadas, en el presente trabajo presentamos una sistematización del uso de estos recursos en el ámbito del aula. Esta experiencia didáctica tiene como objetivo analizar los resultados de aprendizaje del alumnado de segundo año de bachillerato al usar el cine como una herramienta metodológica en las clases de Historia del Arte. El professor Miquel Porter i Moix ha establecido tres modos en los que el cine puede aprovecharse: educando en, con y para el cine, enmarcándose el valor del cine para el aprendizaje de la materia histórica dentro del segundo nivel (Valero, 2008, p.167; Porter, 1983). Entonces, el cine es un recurso que, difícilmente, podría ser empleado en una clásica clase magistral y, por lo tanto, incorporarlo en nuestro día a día supone una renovación de la estructura de esta. 


\section{Aproximación teórica}

En el caso de la asignatura de Historia del Arte hemos de destacar que los estudios más generales sobre las relaciones entre Cine e Historia suelen hacer referencia a contenidos artísticos, al tener presente que la imagen cinematográfica no supone una representación fiel de la realidad, sino una representación subjetiva que el director de cine interpreta de la vida de los artistas y sus obras. Desde los años 30 del siglo pasado, el cine encontró un filón en las vidas de los más famosos artistas de la época moderna y contemporánea, por ejemplo, Rembrandt de Alexander Korda (1936) o The Moon and Sixpence (1943), película de Albert Lewin inspirada en la figura de Paul Gauguin. Desde aquel entonces, las vidas de muchos artistas han sido objeto de versión cinematográfica aprovechándose de modas, homenajes o conmemoraciones históricas para convertirlas en auténticas máquinas de hacer dinero (Ríos \& Escalera, 2014, p. 67).

Asimismo, existe gran cantidad de filmes a los que se les puede sacar partido en las clases de Historia del Arte. El cine puede emplearse en las clases de Historia del Arte para presentar conceptos o épocas; profundizar en la vida y obra de un artista; ilustrar el lenguaje artístico de determinado artista y explicar una técnica determinada como puede ser el grabado o el dripping (Madrid, 2015, p. 60). Destacan, también, las biografías históricas, películas en las que se desarrolla la vida de un individuo relevante y la relación con su entorno. El biopic o biographical picture es una de las tipologías más recurridas dentro del cine de tipo histórico. En estas películas se recrea la vida de personajes históricos y tienen una gran utilidad didáctica en las asignaturas relacionadas con Historia, ya que a través de ellas los alumnos y alumnas pueden acceder al personaje de una forma más rápida (Camarero, 2008).

En el caso de la Historia del Arte, existe una gran cantidad de biopics sobre grandes figuras, como son El tormento y el éxtasis (1965), Caravaggio (1986), El Greco (1966 y 2007), La ronda de noche (2007), Los fantasmas de Goya (2006), Mr. Turner (2014) o El loco del pelo rojo (1956) entre una larga lista. En alguna de ellas se recrea por completo la vida del artista. Mientras que otras, tan solo se centran en un determinado periodo. Este tipo de películas son un gran instrumento para que los alumnos comprendan al artista más allá de su fama o sus obras. Así, pueden ver a la persona de carne y hueso (Madrid, 2015, p. 62). Sin embargo, hay biopics cuyo valor no reside en el tratamiento de la figura del artista, sino más bien en cómo interpreta el entorno y la realidad histórica en la que se desenvolvió, lo que nos ayuda a enriquecer la explicación de los temas transversales tratados en la asignatura. Por último, podemos mencionar la recreación o ficción histórica, término acuñado por el cineasta galo Jean-Pierre Comolli (Caparrós, 2007, p. 33), que refiere a filmes cuyo argumento ficticio posee o evoca una verdad o pasaje histórico en su fondo (Díaz, 2013, p. 322). Estas películas son una fuente de interés para el historiador y el alumnado, por la idealización del pasado que se suele realizar. 
Actualmente, el cine es reconocido como una herramienta de investigación y enseñanza, con lo cual, progresivamente, se ha ganado un lugar en el aula, a pesar de la existencia de personas reticentes a su aplicación docente en los diversos ámbitos educativos (Blay, 2014, p. 3). En España, el promotor de los estudios sobre la relación existente entre el Cine y la Historia fue Ángel Luis Hueso, autor de El Cine y el siglo XX (Blay, 2014, p. 6). Hemos de destacar, también, el papel desarrollado por José María Caparrós Lera, fundador y director del Centre d'Investigacions Film-Història en1983, de la Universidad de Barcelona (Rodríguez, 2014, p. 569), el cual afirma que "el filme es una fuente instrumental de la ciencia histórica, ya que refleja, mejor o peor, las mentalidades de los hombres de una determinada época. Además ... las películas pueden ser un medio didáctico para enseñar Historia" (Caparrós, 2004).

\section{Metodología didáctica en la experiencia}

El tratamiento que el cine ha de recibir en el aula no ha de ser el de mero complemento del temario de la asignatura Historia del Arte en segundo año de bachillerato, ya que por lo general, el uso del cine de contenido histórico suele limitarse al de fuente de información bajo el paraguas de una metodología basada en la exposición. Por el contrario, el uso del cine en las aulas ha de estar en concordancia con los contenidos y diseño curricular del centro en el que impartamos la asignatura (Pérez, 2010, p. 6). Podemos emplear una metodología basada en el aprendizaje por descubrimiento, el cual es un instrumento que se ajusta correctamente para insertar el método hipotético deductivo de investigación de la Historia de Arte (Acosta, 2015, pp. 49-50). En lo que refiere a la planificación, llevaremos a cabo un visionado previo de las películas con la finalidad de elaborar materiales y recoger información acerca de las mismas, con el fin de ofrecer a los alumnos una ficha técnica sobre cada una de ellas, además de material complementario para trabajarlas (Díaz, 2013, p. 329).

A la hora de llevar el cine al aula hay que tener en cuenta la disponibilidad o falta de medios y la correcta administración del tiempo disponible para la proyección de la película. También, podemos jugar con diversas propuestas metodológicas como son las siguientes: proyección integra del filme a la totalidad de los estudiantes: a menos que la película tenga una gran calidad y fidelidad histórica, hemos de asumir que no se puede proyectar un film completo en una sesión de 55 minutos. Proyección de una selección de los contenidos más interesantes o secuencias representativas del filme: esto debe realizarse salvaguardando la coherencia del discurso y puede ser una muy buena opción, pues esta fragmentación puede contribuir al aumento de la atención por parte del alumnado en los hechos más interesantes. Particularización e individualización de la visualización de la película: el visionado 
por parte del estudiante en casa puede resultar también beneficioso, se puede habilitar un aula virtual para que el alumno acceda a las películas recomendadas y verlas, así, de forma completa, sin restricción de tiempo y espacio.

En lo que respecta al trabajo con los filmes, dependiendo de la relevancia y calidad de la película para el proceso educativo, podemos hacer que los alumnos trabajen de forma individual, visionando en casa, o bien trabajar con grupos reducidos las secuencias más significativas de la película, seleccionadas de antemano, siempre que estas mantengan una destacable relación con la lección en curso (Calero \& Moreno-Vera, 2017, p.10). Como nos referimos arriba, hemos de procurar que todas las escenas guarden relación con los hechos históricos concretos, buscando que el alumno se ponga en la piel del artista o se sienta inmerso en la situación de este.

\section{Participantes en la experiencia didáctica}

En lo que respecta a las participantes en el experimento de innovación didáctica, debemos destacar que en él han tomado parte las nueve alumnas matriculadas en la asignatura Historia del Arte de segundo año de bachillerato del I.E.S. Azorín de Petrer, situado en la zona fronteriza que limita con Elda (en la provincia de Alicante, España). Las edades que comprende este nivel educativo se encuentran entre 17 y 18 años de edad. Estas dos poblaciones forman una conurbación de unos 90.000 habitantes. El alumnado que acude al centro presenta una gran diversidad social y socioeconómica, al tratarse de estudiantes de clase media y que representan un amplio espectro cultural que convive tanto en el centro como en el pueblo. Hemos de tener en cuenta que nuestras alumnas son ya nativas digitales, por lo que no han tenido ninguna dificultad a la hora de acceder a los recursos que les hemos ofrecido, con lo que fomentamos, al mismo tiempo, su competencia digital.

\section{Propuesta didáctica}

Los objetivos didácticos que se pretenden alcanzar mediante la utilización de la película Mr. Turner (2014) en la asignatura de Historia del Arte son los siguientes: aprender, a través del cine, las principales características del Romanticismo británico. Comprobar si el cine, como herramienta didáctica, presenta una utilidad notable a la hora de hacer comprender a los estudiantes la personalidad y estilo artístico de Joseph Mallord William Turner. Consolidar los contenidos de la asignatura a través de la visualización de películas estrechamente relacionados con la materia. Contrastar si la imagen o los hechos relatados en el film Mr. Turner (2014) se ajustan a la realidad histórica o si dicha película refleja correctamente el periodo histórico en el que se sitúa la acción, es decir, la Gran Bretaña de comienzos y mediados del siglo XIX. Distinguir o identificar entre los hechos reales y la 
ficción con el fin de sacar el máximo provecho a la visualización del film Mr. Turner (2014). Mostrarse crítico ante la ficción histórica apoyándose en los conocimientos impartidos en la asignatura.

\section{La figura de J.M.W. Turner a través de la película Mr. Turner}

En lo que respecta al Romanticismo británico, emplearemos la película Mr. Turner (2014), dirigida por el británico Mike Leigh y protagonizada por Timothy Spall. Esta refleja la vida de pintor británico Joseph Mallord William Turner, uno de los genios más destacados de la pintura británica de la primera mitad del siglo XIX. En el filme se muestra, tanto la vida personal como la carrera artística de Turner (Figura 1). En el ámbito personal, la muerte de su padre, el barbero y peluquero William Turner, que servía como sirviente y preparador de los colores en la casa de su hijo (Bockemühl, 2004, p. 9), marca un antes y un después en la vida del pintor, quien mantiene una relación compleja con su sirvienta y hace caso omiso a las peticiones de su exmujer (Segura, 2015, p. 88). Este filme resulta muy interesante para su visualización por parte del alumnado debido a que refleja tanto la evolución artística de Turner como los entresijos del mercado del arte en la Gran Bretaña de comienzos del siglo XIX, al destacar el papel de la Royal Academy o del crítico John Ruskin, uno de sus más fieles seguidores, a pesar de la caricatura que la película realiza de este personaje. También es digna de mención la aparición de la científica Mary Somerville, la "reina de las ciencias del siglo XIX" (Segura, 2015, p. 91).

Figura 1. Joseph Mallord William Turner llevando a cabo la obra "El barco de guerra Temeraire remolcado a dique seco para ser desguazado" 1838.

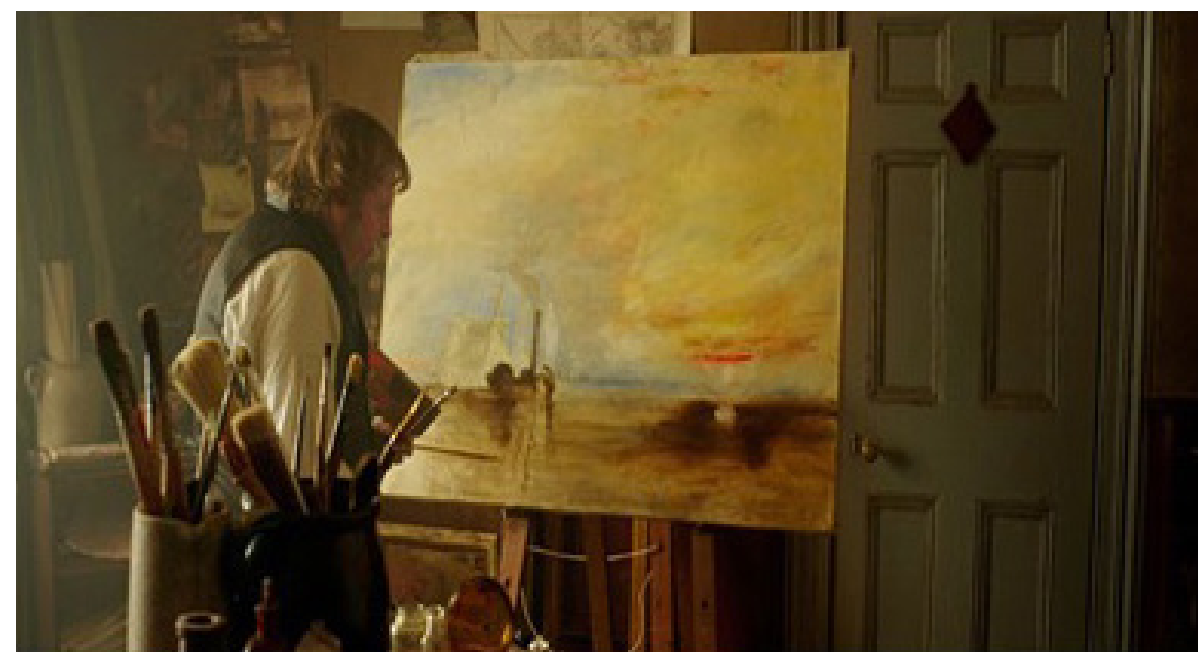

Fuente: Aguilar (2015) 
Entre las obras que aparecen en el film hemos de citar: Lluvia, vapor y velocidad. El gran ferrocarril del Oeste (1844), El barco de guerra Téméraire remolcado a dique seco para ser desguazado (1839), Tormenta de nieve en el mar (1842), Tormenta de nieve: Aníbal cruzando los Alpes (1812), Barco de esclavos (1840) también conocido como Negreros echando por la borda a los muertos y moribundos, y El gran canal de Venecia (1835). Estas obras citadas, o bien aparecen durante su proceso de ejecución, como Lluvia, vapor y velocidad. El gran ferrocarril del Oeste (1844), El temerario remolcado a dique seco (1839), o bien aparecen finalizadas como es el caso de Aníbal cruzando los Alpes (1812) o Negreros echando por la borda a los muertos y moribundos (1840). También, es digno de mención que el filme a modo de tableau vivant recrea una obra menos conocida del autor, Petworth: el artista y sus admiradores (1830), en la que aparece el propio pintor llevando a cabo una pintura bajo la atenta mirada de tres jóvenes muchachas. Otro aspecto que hemos de mencionar es como la película presenta los viajes realizados por el pintor para hallar la inspiración para sus obras. Así, se muestra en ellas el impacto de la revolución industrial y los nuevos tiempos sobre la campiña inglesa.

\section{Evaluación}

En lo que respecta a la evaluación de la eficacia de la innovación docente, es decir, en este caso a la utilización de películas para consolidar los contenidos impartidos en la asignatura de Historia del Arte, hemos entregado a las alumnas un cuestionario sobre la película Mr. Turner (2014) de Mike Leigh. La actividad tuvo como objetivo que, tras el visionado contesten a una serie de preguntas sobre los elementos más importante en los que las alumnas debían focalizar su atención (Díaz, 2013, p. 329). En el caso de esta película, debido a su calidad y relación con los contenidos, hemos optado por la particularización e individualización de la visualización por parte de las alumnas, para que así pudieran estar concentradas en todos los aspectos de la película, le sacasen un mayor partido y no estuviesen concentradas en responder a las preguntas planteadas en el cuestionario. Hemos optado por este modo de visualización con el objetivo de que las respuestas no estuviesen condicionadas por las del resto de compañeras.

En lo referente a los aspectos tratados por el cuestionario nos hemos centrado en cómo la película trata los siguientes tres aspectos: la personalidad de Joseph Mallord William Turner; el arte y el estilo de su pintura; y el mercado del arte en la Gran Bretaña del siglo XIX. En el campo de la personalidad hemos planteado a las alumnas cuestiones sobre la imagen que la película transmite del pintor, su relación con los personajes aparecidos en el filme, tales como su padre, su ama de llaves o su exmujer. En lo que respecta al arte y al estilo de su pintura, las cuestiones han ido dirigidas a la forma, color, contenido, método y temática de la misma. Finalmente, en el mercado del arte, hemos planteado cuestiones 
relacionadas con los espacios de venta, sus relaciones con la Royal Academy of Arts, con los pintores John Constable o Benjamin Haydon, el contexto histórico en el que se desarrolla la cinta, de gran importancia a la hora de contextualizar la obra del pintor en ejercicios como el comentario de obra de arte o las preguntas sobre el contexto histórico que se realizan en los exámenes de acceso a la universidad, los cuales son ejercicios fundamentales en dicha prueba. El objetivo de las preguntas planteadas en el cuestionario es que los puntos anteriores queden claros al alumnado para que este pueda emplearlas en la pequeña biografía del autor que se ha de incluir en los comentarios de obra de arte si son de un autor conocido; el análisis de las características formales y la temática de la obra en un comentario de obra de arte; el contexto histórico que se ha de incluir en un comentario de obra de arte o que se ha de explicar como pregunta independiente. En este marco se han de entender las preguntas relacionadas con el mercado del arte y el papel de la Royal Academy. Además, de una pregunta teórica sobre la pintura del Romanticismo británico, en el que destacan las figuras de J.M.W. Turner y John Constable.

El cuestionario fue entregado a las alumnas junto con una ficha técnica (Figura 2) sobre el filme en el que se especificaban datos como la dirección, producción, intérpretes, entre otros, así como una sinopsis. El cuestionario se entregó en una tabla (Tabla 1) igual a la que se presenta acontinuación. 
Figura 2. Mr. Turner.

\begin{tabular}{|c|c|}
\hline \multicolumn{2}{|c|}{ Ficha Técnica } \\
\hline 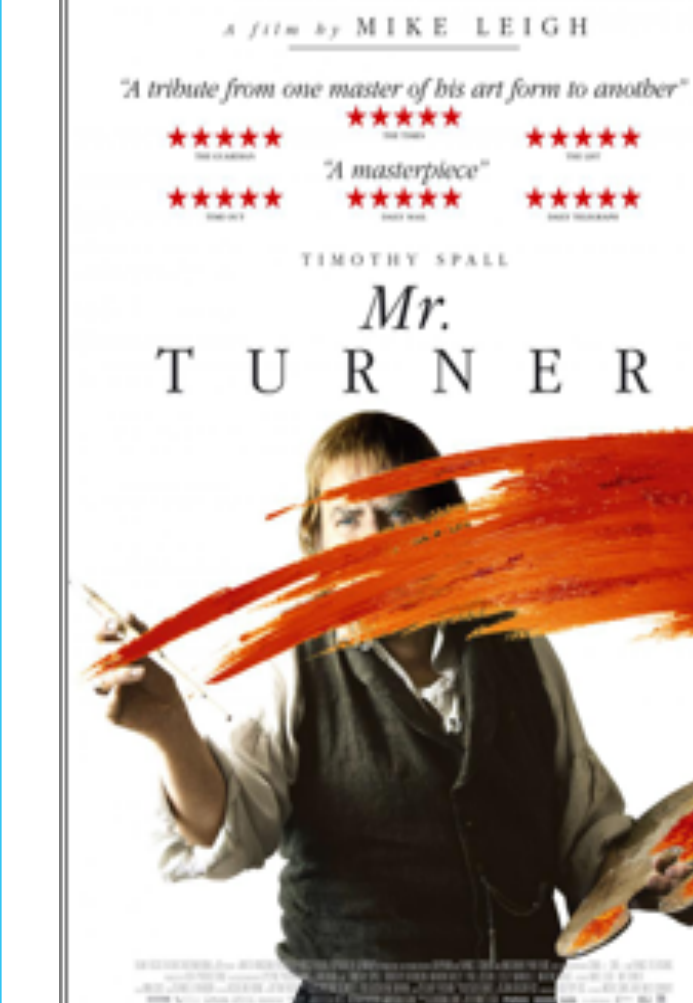 & $\begin{array}{l}\text { Lugar: } \\
\text { Reino Unido-Alemania-Francia, } 2006 . \\
\text { Dirección: } \\
\text { Mike Leigh. Guión: Mike Leigh. } \\
\text { Producción: } \\
\text { British Film Institute, Focus } \\
\text { Features International, Film4, Thin Man Films, } \\
\text { Xofa Productions, Lipsync Productions. } \\
\text { Fotografía: } \\
\text { Dick Pope. } \\
\text { Música: } \\
\text { Gary Yershon. } \\
\text { Vestuario: } \\
\text { Yvonne Blake. } \\
\text { Montaje: } \\
\text { Jon Gregory. Duración: } 149 \text { minutos. }\end{array}$ \\
\hline $\begin{array}{l}\text { Intérpretes: } \\
\text { Timothy Spall (Joseph Mallord William Tu } \\
\text { (Sra. Booth), Paul Jesson (William Turner), } \\
\text { Somerville), Ruth Sheen (Sarah Danby), F } \\
\text { Fleet (John Constable), Joshua McGuire (. } \\
\text { Sinopsis } \\
\text { El largometraje, dirigido por Mike Leigh, y } \\
\text { fica la vida del pintor británico Joseph Ma } \\
\text { británicos del siglo XIX. Este artista fue un } \\
\text { muy buenas relaciones con la aristocracia } \\
\text { de los viajes que realizó a lo largo de Gr } \\
\text { entre dos mundos. Al mismo tiempo, el fil } \\
\text { gados o con destacables figuras del arte } \\
\text { crítico de arte John Ruskin. }\end{array}$ & $\begin{array}{l}\text { Dorothy Atkinson (Hannah Danby), Marion Bailey } \\
\text { Savage (Benjamin Haydon), Lesley Manville (Mary } \\
\text { shton-Griffiths (Henry William Pickersgill), James } \\
\text { uskin). } \\
\text { onizado por Timothy Spall, trata de forma biográ- } \\
\text { illiam Turner, uno de los más destacados artistas } \\
\text { miembro de la Royal Academy of Arts y mantuvo } \\
\text { obra destaca la búsqueda de inspiración a través } \\
\text { aña y como esta refleja una etapa de transición } \\
\text { eja las relaciones que mantuvo con sus más alle- } \\
\text { co como John Constable, Benjamin Haydon o el }\end{array}$ \\
\hline
\end{tabular}

Fuente: Elaboración propia 
El cine para enseñar y aprender Historia del Arte.

La pintura de William Turner

Tabla 1. Cuestionario sobre la película Mr. Turner

\begin{tabular}{|c|c|c|c|}
\hline \multicolumn{4}{|c|}{ Personalidad } \\
\hline $\begin{array}{l}\text { La película refleja a William } \\
\text { Turner como una persona: }\end{array}$ & Agradable y empática & Agresiva y egoísta & Inteligente y solitario \\
\hline Su relación con su padre: & Muy importante & Normal & No tienen relación \\
\hline $\begin{array}{l}\text { Su relación con los demás } \\
\text { personajes es: }\end{array}$ & Fluida & Compleja & Difícil \\
\hline $\begin{array}{l}\text { Su relación con su exmujer y } \\
\text { sus hijas es: }\end{array}$ & Fluida & Compleja & Difícil \\
\hline \multicolumn{4}{|c|}{ Arte y estilo de su pintura } \\
\hline La pintura de Turner es: & Figurativa & Abstracta & Simbolista \\
\hline $\begin{array}{l}\text { Los colores que predominan en } \\
\text { la obra de Turner son: }\end{array}$ & Blanco y negro & Los colores pastel & Los colores vivos \\
\hline $\begin{array}{l}\text { En las obras de Turner } \\
\text { predominan: }\end{array}$ & Las líneas & $\begin{array}{l}\text { Los contornos di- } \\
\text { fuminados }\end{array}$ & El puntillismo \\
\hline $\begin{array}{l}\text { La película muestra a Turner } \\
\text { como un pintor: }\end{array}$ & $\begin{array}{l}\text { Metódico, tiene pensa- } \\
\text { da la obra de antema- } \\
\text { no y todas sus partes. }\end{array}$ & $\begin{array}{l}\text { Caótico, la obra va } \\
\text { adquiriendo forma a } \\
\text { medida que es eje- } \\
\text { cutada por el autor. }\end{array}$ & $\begin{array}{l}\text { Que realiza sus pin- } \\
\text { turas en función de } \\
\text { las modas, sin te- } \\
\text { ner un estilo propio. }\end{array}$ \\
\hline $\begin{array}{l}\text { Turner halla la inspiración para } \\
\text { su obra en: }\end{array}$ & La literatura & La naturaleza & $\begin{array}{l}\text { Pinta temas imagi- } \\
\text { narios }\end{array}$ \\
\hline La obra de Turner refleja: & $\begin{array}{l}\text { Paisajes de la campi- } \\
\text { ña inglesa }\end{array}$ & $\begin{array}{l}\text { Leyendas y héroes } \\
\text { medievales }\end{array}$ & $\begin{array}{l}\text { El impacto de la re- } \\
\text { volución Industrial }\end{array}$ \\
\hline
\end{tabular}

ESCENA. Revista de las artes, 2019, Vol. 79, Núm. 1 (julio-diciembre), pp. 99-118 


\begin{tabular}{|l|l|l|l|}
\hline \multicolumn{2}{|c|}{ Mercado del arte } \\
\hline $\begin{array}{l}\text { Refleja la película los diversos } \\
\text { modos mediante los cuales } \\
\text { vendía los pintores su obra: }\end{array}$ & Exposiciones privadas & $\begin{array}{l}\text { Exposiciones en } \\
\text { salones públicos }\end{array}$ & Encargos privados \\
\hline $\begin{array}{l}\text { La relación de Turner con John } \\
\text { Constable según la película es: }\end{array}$ & Buena & Cordial & Mala \\
\hline $\begin{array}{l}\text { La relación de Turner con los } \\
\text { miembros de la Royal Academy } \\
\text { of Arts es: }\end{array}$ & Buena & Cordial & Mala \\
\hline $\begin{array}{l}\text { La relación de Turner con Ben- } \\
\text { jamin Haydon está marcada } \\
\text { por: }\end{array}$ & La influencia artística & $\begin{array}{l}\text { Los prestamos } \\
\text { económicos }\end{array}$ & $\begin{array}{l}\text { Ser compañeros } \\
\text { de la Academia }\end{array}$ \\
\hline $\begin{array}{l}\text { La trama de la película se desa- } \\
\text { rrolla en: }\end{array}$ & El siglo XVIII & El siglo XIX & El siglo XX \\
\hline
\end{tabular}

Fuente: Elaboración propia.

\section{Discusión y resultados}

A continuación, se procederá a discutir los resultados obtenidos en las encuestas tras llevarse a cabo el visionado del film Mr. Turner (2014) por parte de las alumnas con las que se ha llevado a cabo el experimento de innovación didáctica. Las variables o ítems seleccionados en esta evaluación responden a resultados en el plano holístico de la obra de arte, donde se entiende que el conocimiento y aprendizaje de la historia del arte no se limita a la mera memorización de datos técnicos y formales con respecto a los estilos artísticos. Sino que, aspectos contextuales (el autor, su vida, su personalidad, entre otros.) y del mercado del arte (los compradores, la academia y sus valores, los mecenas, las instituciones que financian el arte, entre otros) también deben formar parte de la enseñanza de la historia del arte. Debido a que son elementos clave para comprender el desarrollo de los estilos y de los artistas, aunque rara vez en la enseñanza formal se hace hincapié en este tipo de cuestiones. 
Gráfico1. Resultados de los cuestionarios sobre la personalidad de Turner

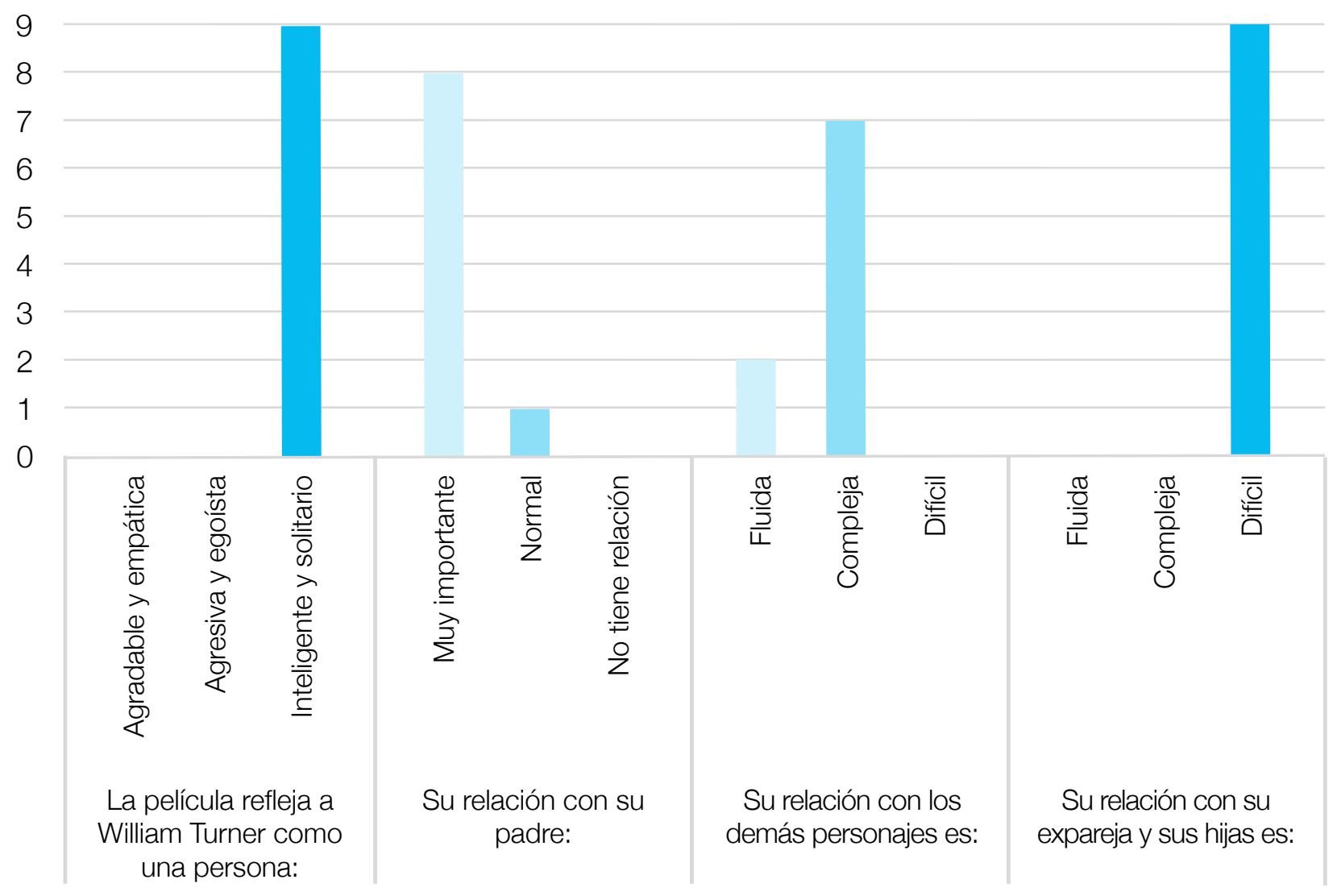

Fuente: Elaboración propia

En lo referente a la personalidad de Joseph Mallord William Turner (Gráfico 1) el $100 \%$ de las participantes han coincidido en indicar que el film muestra al pintor como un personaje inteligente y solitario. La relación con su padre también parece quedar bastante clara a través de la película, ya que ocho de las nueve alumnas han indicado que la relación padre e hijo es muy importante, mientras que una ha indicado que la relación es normal. En lo referente a su relación con el resto de los personajes del film la mayoría de las alumnas, siete de las nueve consultadas, es decir el $77,7 \%$, han indicado que se trata de una relación compleja. Mientras que, las dos alumnas restantes han considerado que es una relación normal. Por último, dentro de este campo, la totalidad de las alumnas han coincidido en indicar que las relaciones entre Turner, su exmujer y sus hijas eran difíciles. 
Gráfico 2. Resultados de los cuestionarios sobre arte y estilo de la pintura de Turner

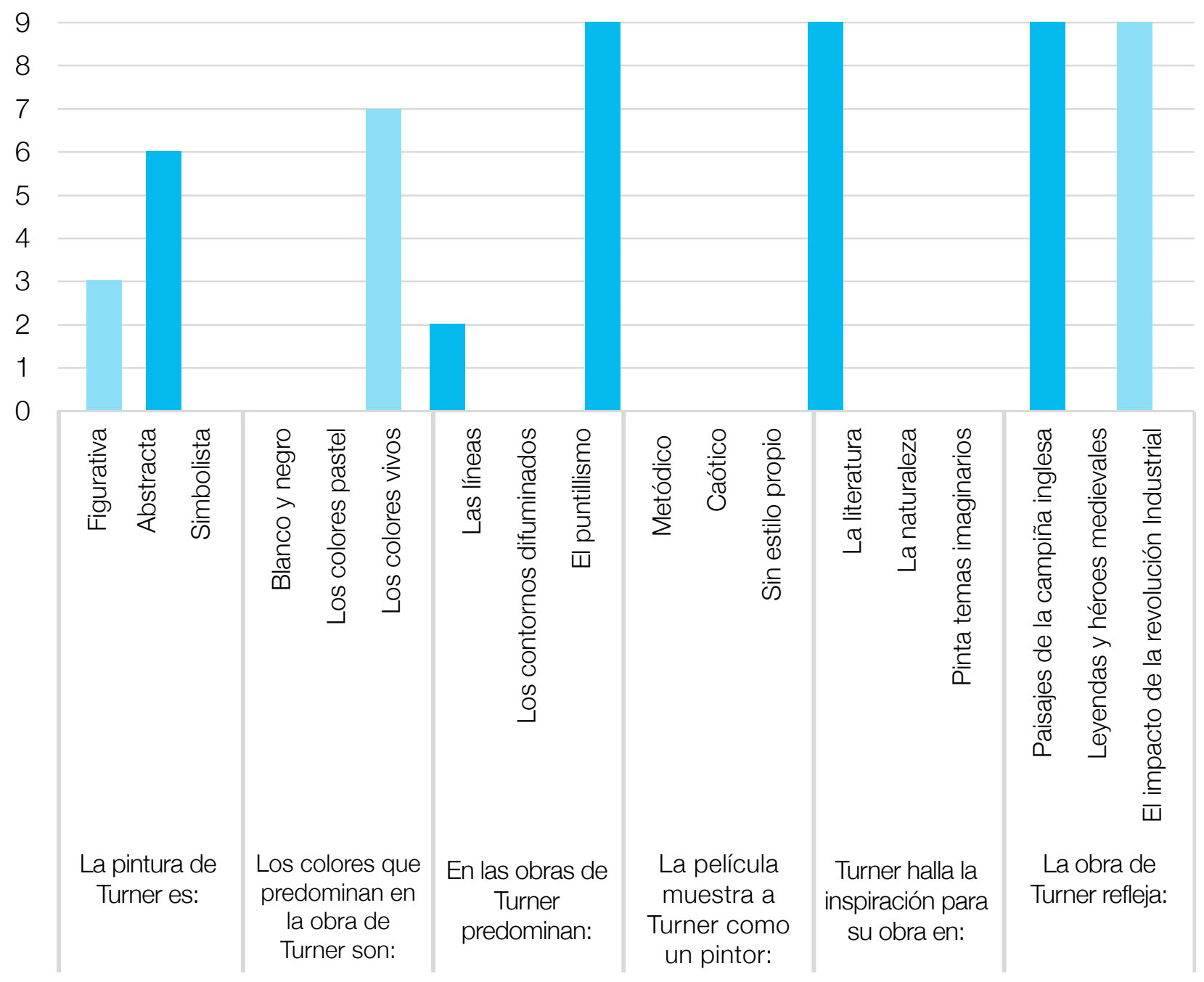

Fuente: Elaboración propia

Por otro lado, en lo que respecta al arte y estilo de la pintura de Joseph Mallord William Turner (Gráfico 2), tres de las alumnas respondieron que su pintura es principalmente figurativa, lo que supone el 33,33\% mientras que las otras seis, el 66,66\% han indicado que es de tipo abstracto. A pesar de la difusión de los contornos, la pintura de Turner no pierde nunca la referencia del natural y de la figuración. 
En lo que respecta a los colores que predominan en la obra de Turner, siete de las nueve alumnas indicaron que los colores pastel son los predominantes. Mientras que, las otras dos alumnas indicaron los colores vivos como los predominantes en su obra. En lo referente a la forma de su obra, la totalidad de las alumnas han señalado que, frente a la línea o el puntillismo, los contornos difuminados predominan en la obra del pintor inglés, lo que supone que el 100\% de las estudiantes ha sabido identificar correctamente el estilo del pintor. Por otro lado, la cuestión sobre cómo muestra la película a Turner y en dónde halla la inspiración para su obra, de nuevo la totalidad de las alumnas coincidieron en indicar que el filme muestra a Turner como un pintor caótico que halla la inspiración en la naturaleza a través de sus viajes. La última pregunta de este apartado es una de las que ha generado una cierta confusión, ya que a pesar de que todas las alumnas escogieron que la obra de Turner refleja paisajes de la campiña inglesa, hubiera sido más acertado que escogieran el impacto de la revolución industrial. Aunque es cierto que para Turner los avances de la revolución industrial se incluyen ya como parte del nuevo paisaje del siglo XIX.

Finalmente, en lo que respecta a las preguntas acerca del mercado del arte (Gráfico 3), estas han generado una mayor variedad de respuestas entre las alumnas. En lo referente a los diversos modos mediante los cuales vendían los pintores su obra, cuatro de las nueve alumnas, es decir el 44,4\%, optaron por las exposiciones privadas, las otras cinco han optado por las exposiciones en salones públicos. Con respecto a la relación de Turner con John Constable, esta pregunta ha sido muy problemática, ya que cinco alumnas han indicado que la relación entre ambos era buena, cuando en realidad era bastante mala, como han indicado las otras cuatro alumnas.

En lo que respecta a la relación de Turner con los miembros de la Royal Academy of Arts, siete alumnas han indicado que era cordial. Mientras que dos, el 22,2\% del total, han señalado que era buena. Por otro lado, la relación de Turner con Benjamin Haydon ha generado también la división de opiniones entre las alumnas, ya que cuatro han indicado los préstamos económicos y cinco han indicado el ser compañeros de la Academia. La última cuestión ha suscitado la unanimidad de las respuestas, pues todas las alumnas han indicado que la trama se desarrolla en el siglo XIX. 
Gráfico 3. Resultados de los cuestionarios sobre el mercado del arte

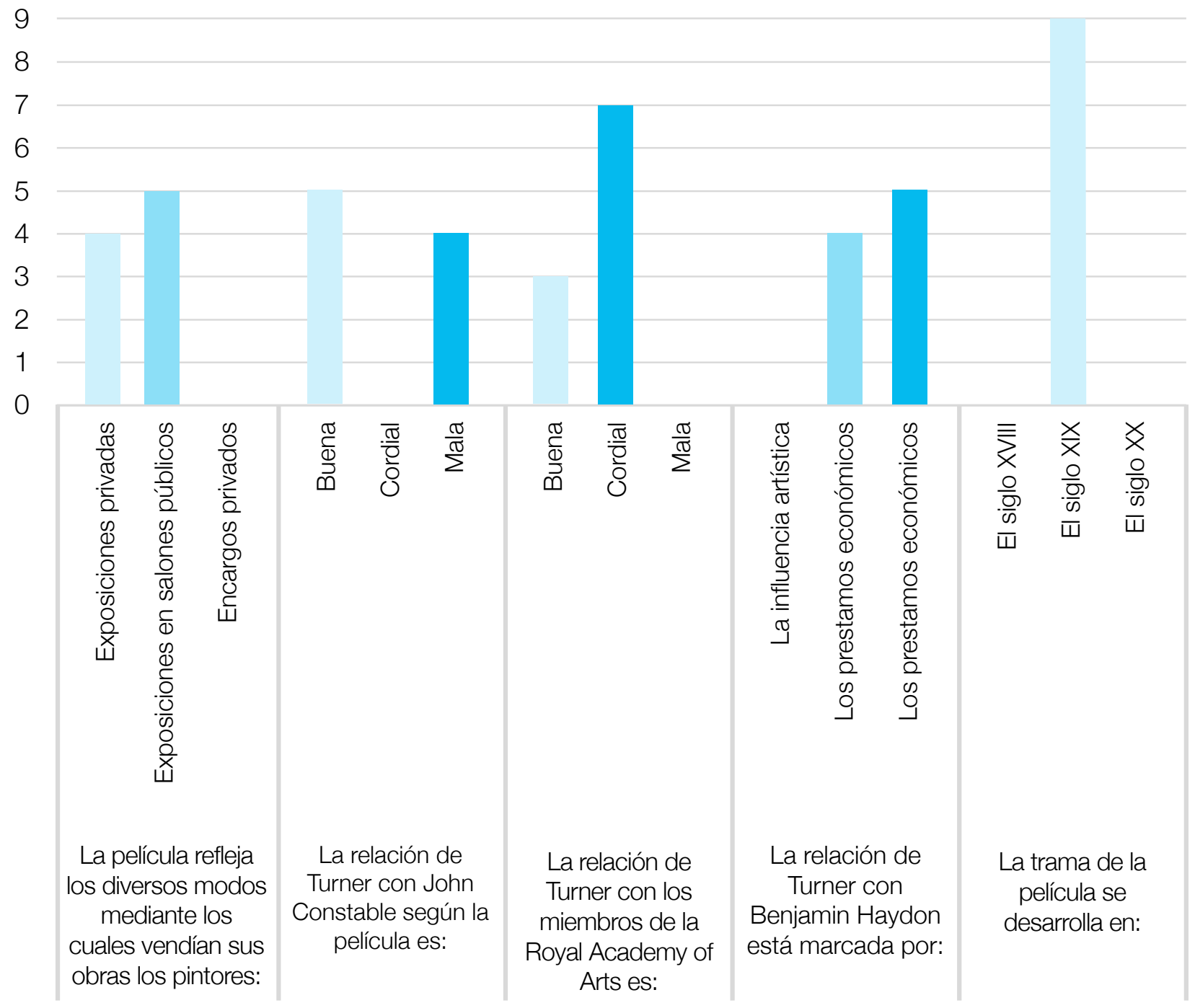

Fuente: Elaboración propia

\section{Conclusiones}

Llegados a este punto, en el cual somos conscientes de la importancia del cine de tipo histórico para la enseñanza y aprendizaje de la Historia, hemos de reflexionar sobre los resultados de la innovación didáctica propuesta en este trabajo. Como hemos podido constatar a través de los cuestionarios realizados por las alumnas sobre la película Mr. Turner 
El cine para enseñar y aprender Historia del Arte.

Artículos

La pintura de William Turner

(2014), la información ofrecida por el filme ha quedado bastante clara, salvo en determinadas preguntas, las cuales como hemos mencionado en el apartado anterior, han generado cierta confusión entre las alumnas.

Por ello, podemos afirmar que, en gran medida, hemos logrado que las alumnas alcancen los objetivos planteados en esta propuesta didáctica como son el aprender a través del cine las principales características de los periodos artísticos u artistas que trabajamos en clase; comprobar si el cine como herramienta didáctica presenta una utilidad notable a la hora de hacer comprender al alumnado los estilos artísticos y las personalidades de los pintores y pintoras trabajados en clase, en este caso, al prestar especial atención a la personalidad y estilo artístico de Joseph Mallord William Turner. En el caso de este pintor, hemos logrado que la mayoría del las alumnas tenga un concepto claro sobre la personalidad, arte y estilo de J.M.W. Turner, ya que las respuestas al cuestionario planteado han sido, mayoritariamente unánimes, lo que evidencia que el alumnado ha entendido a Turner como un genio solitario con complejas relaciones sociales y familiares, cuya pintura difiere en gran medida de la de sus contemporáneos, ya que a pesar de compartir la temática paisajística con otros artistas como Constable, su técnica pictórica se basaba en los contornos difuminados y el empleo de los colores pastel. Las respuestas obtenidas sobre el mercado del arte han sido más variadas, ya que cuestiones como la relación de Turner con Constable $u$ otros miembros de la Royal Academy of Arts han suscitado las más variadas respuestas, lo que requeriría un revisionado por parte del alumnado o una aclaración sobre esto por parte del profesor.

Otro de los objetivos alcanzados fue el consolidar los contenidos impartidos en la asignatura a través de la visualización de películas estrechamente relacionados con la materia y contrastar si la imagen o los hechos relatados en los filmes utilizados se ajustan a la realidad histórica, o si dichas películas reflejan correctamente el periodo histórico en el que se sitúa la acción. Por lo tanto, podemos afirmar que, mediante la visualización del filme Mr. Turner (2014), nuestro alumnado ha conseguido alcanzar un conocimiento más amplio de la figura de J.M.W. Turner en comparación con las metodologías de enseñanzas tradicionales, ya que estas solo nos permiten transmitir conocimientos compartimentados. Mientras que, la utilización de una cinta como Mr. Turner (2014) nos ayuda a contextualizar la vida y obra del pintor y a entender su personalidad, cuestión clave a la hora de comprender su obra que, como representa el filme, era un unicum en la Gran Bretaña de comienzos del siglo XIX. Ello supuso un gran contraste con la metodología magistral que el profesor encargado del grupo empleaba, basada, exclusivamente, en la explicación de contenidos teóricos, y cuyos resultados fueron negativos, tanto en las pruebas teóricas como prácticas. De hecho, en la metodología expositiva tradicional del aula, aspectos como el contexto, la vida del autor, su personalidad, el mercado del arte o su relación con otros pintores nunca aparecieron. Por lo 
que, el uso del cine para abordar la enseñanza de la Historia del Arte permite, al profesorado incluir novedades en los contenidos conceptuales, más allá de los tradicionales elementos formales de las obras.

Las principales dificultades que hemos tenidos que afrontar han sido, sobre todo, los medios técnicos, debido a que las condiciones del centro nos impedían utilizar a menudo los medios de reproducción, por lo que tuvimos que recurrir al visionado individualizado por parte de las alumnas. En lo que refiere a los tipos de visionado, bajo nuestro punto de vista, consideramos, al contrario de lo que afirman diversos autores (Madrid, 2015, p. 59), que el visionado completo de una película puede resultar muy útil para el alumnado. Esto siempre que la película tenga una calidad y adecuación con respecto al temario, que merezca la pena invertir más de una sesión en ella y, además, que el profesor prepare las sesiones para poder trabajar el temario al mismo tiempo (Madrid, 2015, p. 58).

\section{Referencias}

Acosta, L. M. (2015). "Role-playing" basado en cine de género histórico y enseñanza de la historia. Iber: Didáctica de las ciencias sociales, geografía e historia, 80, 48-56.

Aguilar, I. (15 de enero de 2015). Re: Mr. Turner [blog]. Recuperado de http://www.harmonicacinema.com/mr-turner/

Blay, J.M. (2014). "35 mm que cambiaron la historia. El cine como recurso didáctico en la explicación de la historia". Revista Supervisión, 21(33), 1-30.

Bockemühl M. (2004). J. M. W. Turner, 1775-1851: El mundo de la luz y el color. Alemania: Taschen.

Calero, E. \& Moreno-Vera, J.R. (2017). La enseñanza del arte clásico y del arte medieval a través del cine. Making of: cuadernos de cine y educación, 130, 8-14.

Camarero, G. (2008). Una ventana indiscreta: La historia desde el cine. Madrid, España: Ediciones JC.

Caparrós, J. M. (2004). 100 películas sobre historia contemporánea. Madrid: Alianza editorial.

Caparrós, J. M. (2007). Enseñar la historia contemporánea a través de la ficción. Quaderns de cine, 1, 25-35.

Díaz, J. J. (2013). Cine y enseñanza de la historia. En J. J. Díaz Matarranz, A. Santiesteban Fernández, A. Cascajero Garcés (Eds.), Medios de comunicación y pensamiento crítico: nuevas formas de interacción social (pp. 315-333). España: Universidad de Alcalá, Servicio de Publicaciones. 
El cine para enseñar y aprender Historia del Arte.

Artículos

La pintura de William Turner

Madrid, D. (2015). El cine en la didáctica de la historia del arte. Iber: Didáctica de las ciencias sociales, geografía e historia, 80, 57-64.

Pérez, R. (2010). Cine y Educación: explotación didáctica y algunas experiencias educativas. En L. Álvarez, R. Rickenmann y J. Vallés (coords.): // Congrés internacional de didàctiques (pp. 1-6). Girona: Departament de Didàctiques Especifiques. Universitat de Girona

Porter i Moix, M. (1983). El cine como material para la enseñanza de la Historia. En Romanguer, J. y Riambau, E. (Eds.): La Historia y el Cine. Barcelona: Fontamara.

Ríos, S. \& Escalera, R. (2014). El arte en el cine y su uso como ampliación del conocimiento del hecho artístico. El Futuro del Pasado: revista electrónica de historia, 5, 65-89.

Rodríguez, J. (2014). El cine en la realidad de las aulas. Historia y Comunicación Social, 19, 565-574. DOI: https://doi.org/10.5209/rev_HICS.2014.v19.45161

Segura, A. (2015). Mr. Turner: Insulsa desmitificación. FILMHISTORIA Online, 25(1), 88-91.

Valero, T. (2008). Cine e Historia: más allá de la narración. El cine como materia auxiliar de la historia. En G. Camarero (Ed.), I Congreso Internacional de Historia y Cine. Getafe: Instituto de Cultura y Tecnología de la Universidad Carlos III de Madrid, 165-178.

ESCENA. Revista de las artes, 2019, Vol. 79, Núm. 1 (julio-diciembre), pp. 99-118 